\title{
HUMAN VELOCITY AND DIRECTION DISCRIMINATION MEASURED WITH RANDOM DOT PATTERNS
}

\author{
Bart De BruYN* and Guy A. Orban \\ Laboratorium voor Neuro- en Psychofysiologie, Katholieke Universiteit te Leuven, \\ Campus Gasthuisberg, Herestraat, B-3000 Leuven, Belgium
}

(Received 14 December 1987; in revised form 4 May 1988)

\begin{abstract}
In the present experiments three different motion discrimination tasks were studied using a random dot pattern as stimulus: velocity discrimination, direction discrimination and discrimination of opposite directions. The analysis of the motion of random dot patterns is based on motion sensitive mechanisms without the confounding interference of position sensitive mechanisms (Nakayama and Tyler, 1981). Furthermore, since isotropic random dot patterns contain no dominant orientation, a change in the direction of motion does not parallel a change in orientation. Hence the use of a random dot pattern as stimulus allows velocity and direction discrimination to be compared. Human velocity discrimination displays a U-shaped dependence on the stimulus velocity: the JNDs, expressed as Weber-fractions, are minimal for velocities ranging from 4 to $64 \mathrm{deg} \cdot \mathrm{sec}^{-1}$. The Weber-fractions in velocity, determined with a staircase procedure tracking a $84 \%$ correct response level, were about $7 \%$ at the optimal specds. The velocity discrimination curve obtained with the random dot pattern is similar to that obtained with light bars. Human direction discrimination, defined as the smallest difference in direction which can be resolved, also displays a $U$-shaped dependence on the stimulus velocity. Direction discrimination thresholds decrease up to a velocity of $4 \mathrm{deg} \cdot \mathrm{sec}^{-1}$, they then stay at a constant level up to $128 \mathrm{deg} \cdot \mathrm{sec}^{-1}$. Beyond this velocity the thresholds increase again. The mean direction discrimination threshold was $1.8 \mathrm{deg}$ at optimal speeds. Discrimination of opposite directions, determined for the same conditions as those for which velocity and direction discrimination thresholds were determined, was better than the $90 \%$ response level at all speeds. However at low contrast, opposite directions are reliably discriminated only at intermediate speeds. Perceiving a coherent moving random dot pattern is supposed to be based on a cooperation between a large number of local motion detectors. In order to evaluate the importance of detector output pooling, the influence of the size of the pattern and of the presentation time on the three discrimination tasks was measured. The results indicate that the pooling requirements are task dependent. A somewhat larger pooling is required for velocity discrimination than for direction discrimination, whereas for discrimination of opposite directions only a few local motion detectors are involved.
\end{abstract}

Motion Velocity Just noticeable difference Temporal and spatial summation Local velocity vector Cooperation between detectors

\section{INTRODUCTION}

Previous work from this laboratory has shown that human velocity discrimination measured with a moving bar is optimal at intermediate speeds. Indeed just noticeable differences (JND) in speed are smallest (5-7\% Weber fractions) for speeds ranging from 4 to $64 \mathrm{deg} \cdot \mathrm{sec}^{-1}$ and increase at both sides of this optimal range. The present experiments were undertaken to investigate the generality of the proportion that intermediate speeds are optimal for motion perception. As a stimulus we used a random dot pattern. Since its introduction in psychophysics by Julesz (1971), random dot patterns have been

*Research assistant NFWO used extensively to investigate motion sensitivity at the psychophysical level (for review see Nakayama, 1985) as well as at the physiological level (Orban et al., 1975; Hammond and MacKay, 1975, 1977; Allman et al., 1985; Gulyas et al., 1987). There are three reasons why we choose to use a random dot pattern as a stimulus. First of all it has been shown by Nakayama and Tyler (1981) that movement of random dot patterns is detected by motion sensitive and not by position sensitive mechanisms. Hence the use of random dot patterns isolates the short range motion mechanism and makes comparison with physiological results more straightforward since direction selective or velocity tuned cortical cells are considered the neuronal hardware underlying the short range 
mechanism. Second, the random dot pattern we have been using contains equal light and dark parts. Hence smearing of the pattern at fast speeds would produce an homogeneous grey pattern and judgement about fast motion of this random dot pattern cannot be based on the qualities of the image smear such as duration or length as could be the case for a moving bar. Finally a random pattern allows direct comparison between fine discriminations in speed and direction which are the two basic motion parameters.

In the present experiments, three psychophysical performances were measured: JNDs in speed and direction and opposite motion discrimination. Comparison between the JNDs in velocity obtained with a random dot pattern and JNDs in velocity obtained with a bar, should learn how much speed discrimination depends on the stimulus pattern. Comparison between JNDs in speed and direction obtained with a random dot pattern should reveal the differences between the movement detectors underlying both perceptions, assuming that the further processing is relatively similar for both types of JNDs. Our linking hypothesis (Orban, 1985) assumes that the output of velocity tuned cells and of direction-selective cells is used for speed and direction discrimination respectively. We assume as others (Regan and Beverley. 1985) that for JNDs the slope of the tuning curve is the relevant feature of the cell's response property. We also assume that in order to obtain the final sensory representation further processing of the detector's output is required in order to reduce the variability of the slope and the dependency of the slope on other stimulus dimensions. The first requirement follows from the general belief that human JNDs are smaller than the minimum parameter difference that can be detected by single cells, although some have argued the contrary (Parker and Hawken, 1985; Skottun et al., 1987). The second requirement follows from the observation that JNDs do not depend on other parameters than the one to be discriminated, while cortical cell's activity at an early level, depends on many visual parameters. In a previous study we compared velocity discrimination and opposed motion discrimination for a moving light bar (Orban et al., 1984). However these two tests not only differ in visual parameters discriminated but also in psychophysical tasks. Direct comparison between JNDs in speed and JNDs in direction are not possible with a bar as stimulus since direction and orientation are confounded in a moving bar for which the motion axis is orthogonal to the orientation. Since a random dot pattern contains no overall orientation such a confusion will not arise with this pattern. Hence such a pattern allows direct comparison of fine speed and direction discrimination. Comparison between JNDs in direction and opposed motion discrimination will reveal how different the processing of the direction selective cell output is in these two tasks. Since the difference in response of direction-selective cells for opposite directions of motion is large and is much less dependent on other stimulus parameters. one can expect that far less further processing is required for opposed motion discrimination than for fine direction discrimination (JNDs).

In addition to reference velocity, the stimulus speed at which the performances were tested, the size of the pattern and the duration of movement were also manipulated. The study of spatial and temporal summation should reveal the amount of pooling in motion discrimination tasks. Pooling could be part of the further processing which yields the final sensory representation on which the decision is made. It reduces the effect of the variability in the detector's tuning and also, to a certain extent, the dependency of this tuning on other visual parameters. In case of motion discrimination the prediction about pooling depends on the model used for motion detection. Basically two classes of models has been proposed: the gradient model (Marr and Ullman, 1981) and the bilocal correlation model (Hassenstein and Reichardt. 1956; Reichardt, 1961). According to the gradient model a cortical cell can only provide information about the direction of motion and not speed, unless the neuron would know a spatial distance as e.g. its own RF width. Furthermore physiological studies have shown that the direction selectivity and velocity tuning of cortical cells depend on interactions between different loci (Duysens $a t$ al., 1985; Baker and Cynader. 1986). Hence we use here the bilocal correlation model. van Doorn and Koenderink (1982a, b, 1983) have shown that motion detectors tuned to a given velocity can use a range of spans $(\Delta x)$ and a range of delays $(\Delta t)$ since the optimal velocity of the detector equals $\Delta x / \Delta t$. Thus for each velocity we can consider that for each retinal locus there is a pool of detectors luned to a single velocity but covering a range of spans (or delays). Hence 
increasing the size of the randon dot stimulus will have two effects, provided stimulus duration is not limiting: recruiting elements of the pool at a given locus with larger spans and triggering of elements belonging to pools covering different retinal loci. Similarly if one increases the duration of random dot motion one will recruit more detectors from the pool at a given locus and one will also activate the elements of the pool for a longer time. Hence manipulation of size involves both a recruitment from the local pools and a spatial summation across local pools while manipulation of duration involves both recruitment from local pools and temporal integration within the local pool. If temporal and spatial summation effects are mainly due to the recruitment within the local pool the two effects should be proportional and the stimulus velocity should be the proportionality factor.

\section{METHODS}

\section{Subjects}

Three subjects participated in the experiments, HE a corrected hypermetrope and astigmat, WS a corrected myope and BDB an emmetrope. In none of the present experiments a significant difference between the subjects was observed.

\section{Stimulas}

The stimulus used in the experiments was a random dot pattern in which pixels were light (L1) or dark (L2) with equal probability. The pixel size was $0.07 \mathrm{deg}^{2}$. The average luminance $[(\mathrm{LI}+\mathrm{L} 2) / 2]$ was $16 \mathrm{~cd} / \mathrm{m}^{2}$ and the contrast $[(\mathrm{L} 1-\mathrm{L} 2) /(\mathrm{Ll}+\mathrm{L} 2)] 75 \%$. Fourier analysis showed that the energy distribution of the pattern was isotropic, the pattern containing no dominant orientation and that spatial frequencies up to $4 \mathrm{c} / \mathrm{deg}$ were represented in the pattern.

\section{Apparatus}

Movement of the pattern was obtained by turning two mirrors mounted on galvanometers (General Scanning, Inc.) and reflecting the pattern projected by means of a slide projector on to a polacoat screen. Movement of the mirrors was under control of a micro-processor. Presentation by means of a mirror system has, in comparison with the frequently used CRTdisplays. the advantage that one can present a smooth continuous motion. It has the disadvantage that the pattern cannot remain visible throughout the testsequence. Whereas a random dot pattern generated on a CRT display can be refreshed continuously, a pattern projected by means of a slide projector and a mirror system has to move back to the starting position after a movement. Hence a shutter was closed during this return-jump. In order to minimize the on and off transients resulting from this shuttering the pattern was presented stationary for $1 \mathrm{sec}$ before and $1 \mathrm{sec}$ after each motion. As a result the pattern disappeared during the return-jump only after the response of the subject was recorded. Random dot patterns are likely to have areas in which pixels are scanty and other areas in which pixels are more dense. Systematic effects due to uneven spreads in pixels were minimized by randomizing, independently in the $X$ and $Y$ axis, the starting position over a $12 \mathrm{deg}$ rangc. The pattern was only visible in a circular aperture of which the size could be varied. This window had a diameter of $10 \mathrm{deg}$ in all standard conditions. For most reference velocities $\left(0.5-128 \mathrm{deg} \cdot \mathrm{sec}^{-1}\right)$ the mean stimulus duration was $200 \mathrm{msec}$. Due to limitations in the size of the projected image the effective (i.e. taking into account the randomization of start position) maximum movement traverse was $40 \mathrm{deg}$. Larger traverses would have resulted in distortions of the projected imagc. Therefore, larger reference velocities were obtained by decreasing the movement duration. For speed discrimination at a reference velocity of $256 \mathrm{deg} \cdot \mathrm{sec}^{-1}$ movement duration was $150 \mathrm{msec}$. For direction discrimination shorter movement durations had to be used. The digitized ramp function which controlled the mirror drivers reduced to a step function when an extremely slow motion was required. This was the case for the $Y$ component of the motion when the direction of the motion was slightly off the horizontal axis thereby limiting resolution in direction to $5 \mathrm{deg}$. In order to increase this resolution the pattern was moved over a larger extent and the shutter was used to present only that part of the motion, for which the analog driver signal was actually a ramp function. Since the rotation angle of the mirror was limited we had in the direction discrimination experiments to restrict the stimulus duration to $100 \mathrm{msec}$ and $50 \mathrm{msec}$ at 128 and $256 \mathrm{deg} \cdot \mathrm{sec}^{-1}$ respectively. For each reference velocity the movement traverse was fixed and variations in velocity were obtained by small changes in movement duration, except in 
control experiments in which the duration of motion was randomized.

\section{Procedure}

The subjects viewed the pattern binocularly with natural pupils at a distance of $57 \mathrm{~cm}$. Since the motion durations were in the order of the latency of a stimulus-evoked eye movement, the occurrence of these eye-movements was limited (Westheimer, 1954; Haddad and Steinman, 1973). In addition, at least in the velocity discrimination and opposed direction discrimination experiments, motion direction was randomized thereby minimizing the effect of anticipatory eye movements. As was shown by Kowler and Steinman (1979), even if anticipatory eye movements occur they do not predict motion direction consistently. For direction discrimination it has also been shown (Kosnik et al. 1985 ) that the discrimination of the direction of motion is not based on a sensorimotor response. As an aid to maintain fixation, a fixation point $(0.5 \mathrm{deg})$ was presented when the pattern was not moving.

Psychophysical performance was measured for three different tasks: velocity discrimination assessed by measuring the smallest difference in velocity that can be resolved, direction discrimination tested by measuring the smallest difference in motion axis that can be resolved and discrimination between opposite directions on the same axis (opposed direction discrimination). The psychophysical method used to measure JNDs in velocity and direction was a Transformed Up Down (TUD) method (Wetherill and Levitt, 1965: Levitt, 1971). Two stimuli with a given parameter difference, either in velocity or in direction, were presented in sequential order. In the velocity discrimination experiments, the stimuli moved on an horizontal axis and were equally distributed around the reference velocity, $V+D V / 2$ and $V-D V / 2$ where $V$ is the reference velocity and $D V$ the velocity difference. By pressing one of two response keys the subject had to indicate whether the second stimulus moved faster or slower than the first stimulus. In the direction discrimination experiments both stimuli moved at the same velocity. One member of the pair moved from left to right on the horizontal axis, the other member of the pair moved, also from left to right, along an axis rotated clockwise or counterclockwise with respect to the horizontal axis. The subject had to indicate whether the second stimulus moved along an axis rotated clockwise or counterclockwise compared to the motion axis of the first stimulus. Both for speed and direction discrimination the parameter difference was decreased by $20 \%$ after four consecutive correct responses and was increased by $20 \%$ after one incorrect response. Such an algorithm tracks a $84 \%$ correct response level. The TUD method was stopped after 12 reversal points. The geometric mean of the last six reversal points was taken as the threshold. In the opposed direction discrimination experiments the subject had to indicate whether the pattern moved leftwards or rightwards. The pattern moved at the same velocity on the horizontal axis for both directions. The percentages correct responses were determined for 100 stimuli presentations. In all conditions auditory feedback was given after each response. Conditions for each experiment were counterbalanced by testing all conditions twice in random order and finishing the first experimental run of all conditions before testing the same conditions in the second run, in a different random order. The data were analysed using the nonparametric Mann-Whitney U-test (Siegel, 1956).

Whereas no training was needed for opposed direction discrimination, an extensive training had to be given for velocity discrimination as well as for direction discrimination. The training was continued until performance reached a ceiling. The training effects were much larger for velocity than for direction discrimination. Indeed at each speed, about 5-6 daily sessions (each corresponding to the measurement of 1 JND) were required for the direction discrimination, while over 20 daily sessions were required for the velocity discrimination. The improvement was also more substantial for velocity than for direction discrimination. Thresholds only improved by a factor 1.4-1.5 for direction discrimination compared to a factor 2 or 3 for velocity discrimination

Control experiments were run to ascertain that subjects could not use position as a cue for the detection of motion. In these control experiments, the random dot pattern was presented stationary in two positions corresponding to the start and endpoint of a movement but the motion itself was invisible. The interval between both presentations was equal to the standard duration of movement for the different speeds (200 for speeds ranging from 1 to $64 \mathrm{deg} \cdot \mathrm{sec}^{-1}$ and 50 and $150 \mathrm{msec}$ for $256 \mathrm{deg} \cdot \mathrm{sec}^{\prime}$ ). In these conditions the subject was not able to 
discriminate opposite directions of the motion: for all virtual velocities the percentage correct responses was below 60 . One has to note that when opposed motion discrimination is tested in similar conditions with a light bar, it is always perfect (above 90\% correct response level). Since in these control conditions the spatial as well as the temporal intervals are large, this control experiment confirms that the involvement of the long range process (Braddick, 1974) in our experimental conditions was rather limited.

\section{EXPERIMENT 1: VELOCITY DISCRIMINATION MEASURED AS A FUNCTION OF VELOCITY OF THE RANDOM DOT PATTERN}

Just noticeable differences (JNDs) in velocity were measured for velocities ranging from $0.5 \mathrm{deg} \cdot \mathrm{sec}^{-1}$ to $256 \mathrm{deg} \cdot \mathrm{sec}^{-1}$. In Fig. 1 mean data of three subjects are shown. The relationship between JNDs in velocity, expressed as Weber-fractions, and stimulus velocity is $U$ shaped. We refer to this relationship as the velocity discrimination curve. Differential velocity sensitivity improves up to a velocity of $4 \mathrm{deg} \cdot \mathrm{sec}^{-1}$, remains at a more or less constant level of about $7 \%$ for speeds up to $64 \mathrm{deg} \cdot \mathrm{sec}^{-1}$. Beyond this velocity Weber-fractions increase steeply.

In a first control experiment we compared the performance of subjects for trials with fixed durations (implying a correlation between speed and movement traverse) and for trials in which

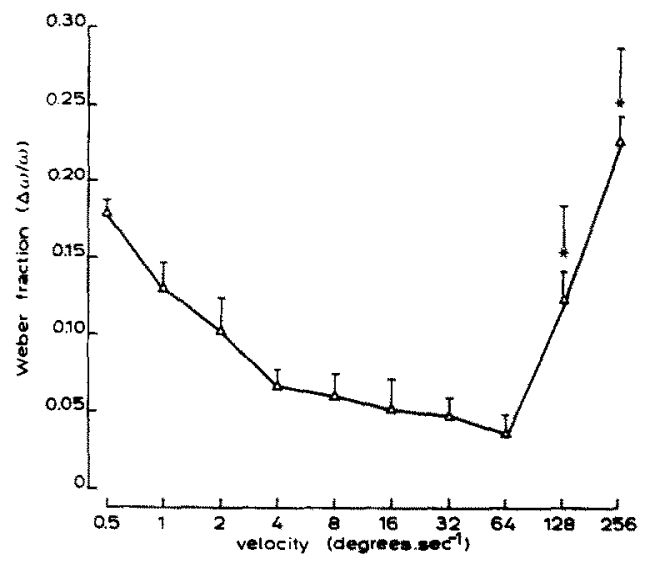

Fig. 1. Just noticeable differences in velocity expressed as Weber-fractions plotted as a function of velocity. Mean data for three subjects (HE, WS and BDB). Vertical bars indicate standard deviations. The points indicated by asterixes are the thresholds measured with shorter stimulus durations than standard, $100 \mathrm{msec}$ for a velocity of $128 \mathrm{deg} \cdot \mathrm{sec}^{-1}$ and $50 \mathrm{msec}$ for a velocity of $256 \mathrm{deg} \cdot \mathrm{sec}^{-1}$. motion duration was randomized. The middle of the range over which duration was randomized was taken as the duration in the fixed duration trials. The results of this comparison are shown in Fig. 2. Although the performance of the subjects was slightly worse when the movement duration was randomized, the differences between both conditions were not significant. It is plain from Fig. 2 that in both conditions the velocity discrimination curve is U-shaped. It is worth noting that the performance of the subjects for the fixed duration condition of Fig. 2 is somewhat poorer than in Fig. 1. This is due to the difference in duration between the stimulus presentations in Figs 1 and 2 and is in keeping with the temporal summation of velocity discriminations which will be described in detail later.

In a second control experiment we sought to rule out differences in contrast sensitivity at different speeds, especially the faster ones, as a cause of differences in differential velocity sensitivity. We matched the contrast of the random dot pattern for the different velocities in the following way. We measured opposed direction discrimination for different contrast levels and different speeds. As shown in Fig. 3A opposed direction discrimination depends on contrast only over a narrow range: a $3 \%$ contrast opposed directions cannot be discriminated at any speed and at $17 \%$ contrast opposed directions can be discriminated at all speeds. From the curves in Fig. 3A we extracted the contrast threshold corresponding to $75 \%$ correct performance for the different speeds. As indicated by

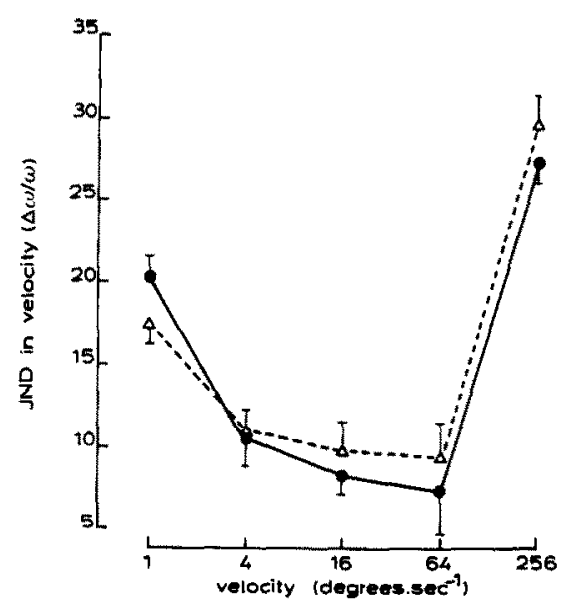

Fig. 2. Comparison between the velocity discrimination curve obtained with fixed duration conditions (full line) and with randomized duration conditions (dashed line). Mean data of three subjects. Vertical bars indicate standard deviations. 

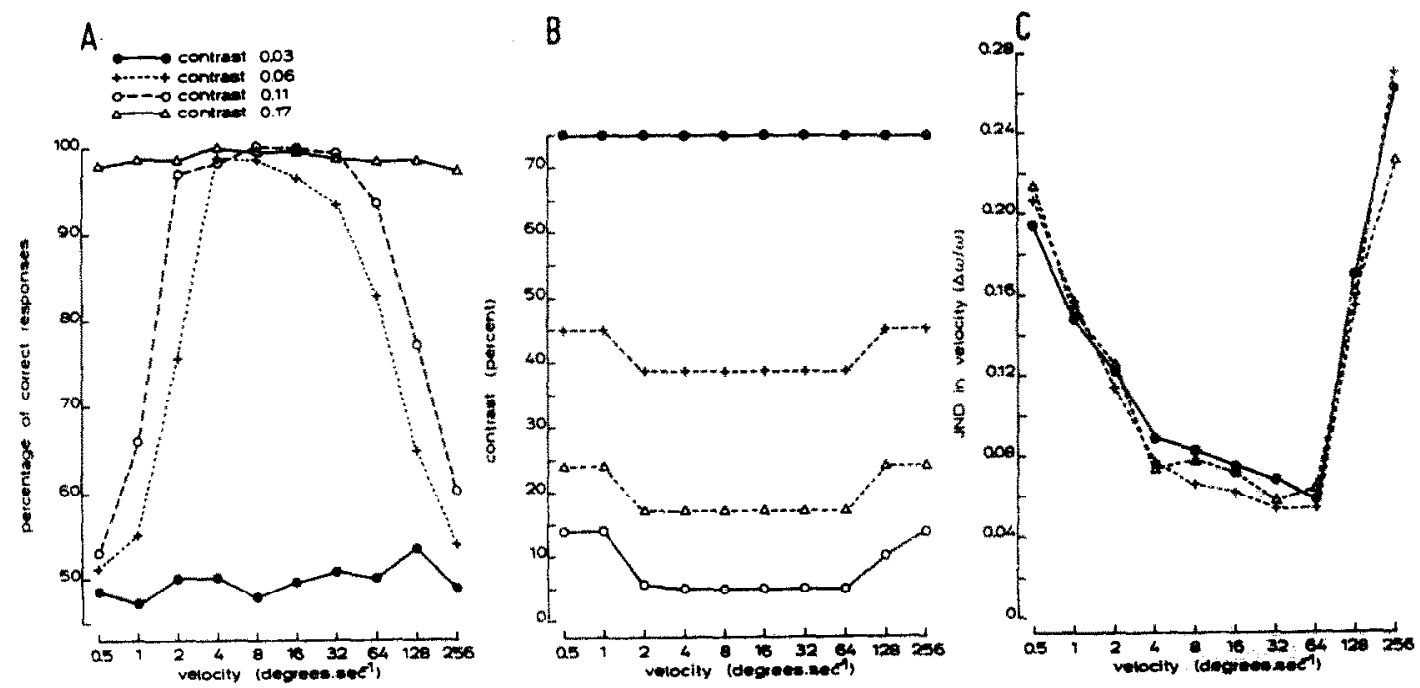

Fig. 3. (A) Opposed direction discrimination measured as a function of stimulus velocity for four different contrast levels. (B) Contrast thresholds corresponding to $75 \%$ correct direction diserimination (open circles), experimental contrast levels set 12 and $32 \%$ above these thresholds (crosses, triangles) and the standard contrast conditions (dots) plotted as a function of stimulus velocity. (C) JNDs in velocity, expressed as Weber fractions, plotted as a function of reference velocity for three different contrast conditions (see 3B).

the circles in Fig. 3B this threshold is about $5 \%$ contrast for speeds between 2 and $64 \mathrm{deg} \cdot \mathrm{sec}^{-1}$ and above $10 \%$ contrast for more extreme speeds. We then selected at the different speeds, the contrasts which were 12 and $32 \%$ above these contrast thresholds (Fig. 3B) and used these values to measure JNDs in velocity. As shown in Fig. 3C the performance was not different in the standard conditions and the conditions in which the contrast was adjusted to the difference in sensitivity at different speeds. Another way to look at the results of Fig. $3 \mathrm{C}$ is to say that for all speeds the performance was independent of the contrast. Hence for all speeds we worked on the plateau of the JND in velocity-contrast curve and thus the difference between the JNDs at different velocities cannot be attributed to a difference in contrast sensitivity.

In order to compare the results obtained with the random dot pattern with the results of our previous studies using a light bar, the stimulus durations for the highest velocities (128 and $256 \mathrm{deg} \cdot \mathrm{sec}^{-1}$ ) were limited to 100 and $50 \mathrm{msec}$ respectively. The results, for those shorter stimulus durations at the higher velocities were compared to the results for the standard conditions in Fig. 1. In the light-bar experiments the stimulus duration at high velocities had to be limited in order to prevent a too parafoveal stimulation. However as shown previously (Orban et al.. 1984) limitation in stimulus duration does not explain the increase in Weberfractions at the high velocities. In Fig. 4 the results of the present experiment $(N=3)$ are compared with the pooled $(N=9)$ results of our previous studies (Orban et al., 1986; De Bruyn and Orban, unpublished). The velocity discrimination curve obtained with the random dot pattern did not differ in a significant way from the one obtained with a light bar $\left(\mathrm{U}_{10,10}=45\right)$. Two out of three subjects who participated in

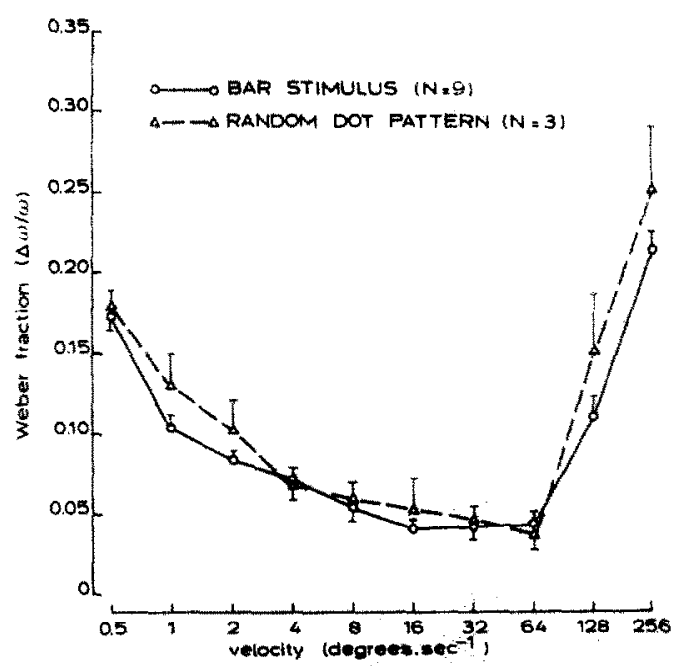

Fig. 4. Comparison between the velocity discrimination curve obtained with a random dot pattern $(N=3)$ and with a moving light bar $(N=9)$. The latter curve is the average of our previous data (Orban et al., 1986; De Bruyn and Orban. unpublished). Vertical bars indicate standard deviations. 
the present experiments did also participate in the earlier studies. Comparison between the data of these two subjects yielded a similar conclusion.

\section{EXPERIMENT 2: DIRECTION DISCRIMINATION MEASURED AS A FUNCTION OF THE VELOCITY OF THE RANDOM DOT PATTERN}

Direction discrimination thresholds, the smallest difference in direction which can be resolved, was measured as a function of velocity for velocities ranging from 0.5 to $256 \mathrm{deg} \cdot \mathrm{sec}^{-1}$. Figure 5 shows the mean data of three subjects. Direction discrimination thresholds decrease linearly with the logarithm of the velocity up to $4 \mathrm{deg} \cdot \mathrm{sec}^{-1}\left(r^{2}=0.98\right)$, they remain at a more or less constant level between 4 and $128 \mathrm{deg} \cdot \mathrm{sec}^{-1}$. Beyond this velocity the differential thresholds increase again. The mean asymptotic direction discrimination threshold for velocities ranging from 4 to $128 \mathrm{deg} \cdot \mathrm{sec}^{-1}$ was $1.8 \mathrm{deg}$ (SD: 0.42).

\section{EXPERIMENT 3: CHANGES IN VELOCITY DISCRIMINATION AND DIRECTION DISCRIMINATION AS A FUNCTION OF THE SIZE OF THE PATTERN}

Velocity discrimination and direction discrimination were measured as a function of the size of the random dot pattern for five velocities: $1,4,16,64$ and $256 \mathrm{deg} \cdot \mathrm{sec}^{-1}$, a representative sample of the range of velocities over which the

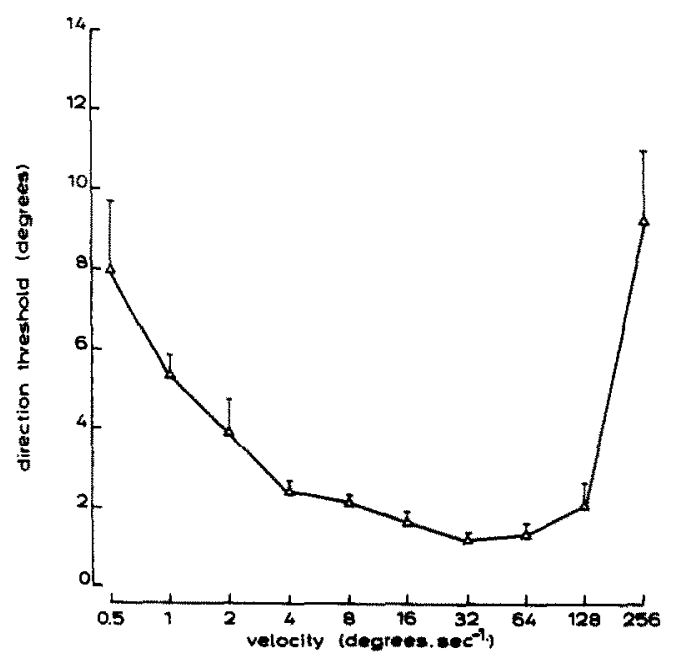

Fig. 5. Direction discrimination thresholds plotted as a function of the velocity of the random dot pattern. Mean data of three subjects. Vertical bars indicate standard deviations. human visual system operates. The area of the random dot pattern was changed by varying the diameter of the circular aperture in which the pattern appeared. At each velocity performance was measured for stimulus diameters ranging from 3 to 20 deg. Figure 6 shows the effect of the size of the pattern on the JNDs in velocity. These results show that only for a velocity of $256 \mathrm{deg} \cdot \mathrm{sec}^{-1}$, JNDs in velocity depend on the area of the stimulus pattern. For this velocity performance improved as the size of the pattern increased up to $20 \mathrm{deg}$ dia. For velocities below $64 \mathrm{deg} \cdot \mathrm{sec}^{-1}$ JNDs in velocity do not depend on the size of the pattern. For a velocity of $64 \mathrm{deg} \cdot \mathrm{sec}^{-1}$, a small increase of the JND in velocity was observed as the size of the pattern was increased from 3 to $5 \mathrm{deg}$.

Figure 7 shows the dependence of the direction discrimination thresholds on the size of the stimulus. As was the case for velocity discrimination, changes in direction discrimination as a function of the size of the stimulus were only observed for a velocity of $256 \mathrm{deg} \cdot \mathrm{sec}^{-1}$. There was little spatial summation effect for velocities ranging from 1 to $64 \mathrm{deg} \cdot \mathrm{sec}^{-1}$. For a velocity of $256 \mathrm{deg} \cdot \mathrm{sec}^{-1}$ the movement duration was $150 \mathrm{msec}$ in the velocity discrimination experiments and only $50 \mathrm{msec}$ in the direction discrimination experiments (see Methods). Therefore we measured as a control, the spatial

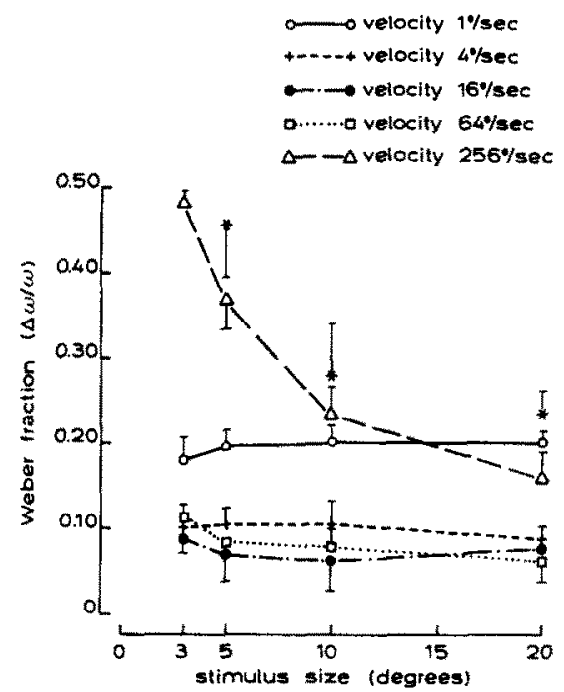

Fig. 6. JNDs in velocity, expressed as Weber-fractions, platted as a function of the size of the random dot pattern. The size of the pattern was changed by varying the diameter of the circular aperture in which the pattern appeared. Mean data for three subjects. The points indicated by the asterixes show the results for a shorter stimulus duration ( $50 \mathrm{msec}$ ) at a velocity of $256 \mathrm{deg} \cdot \mathrm{sec}^{-1}$. Vertical bars indicate standard deviations. 


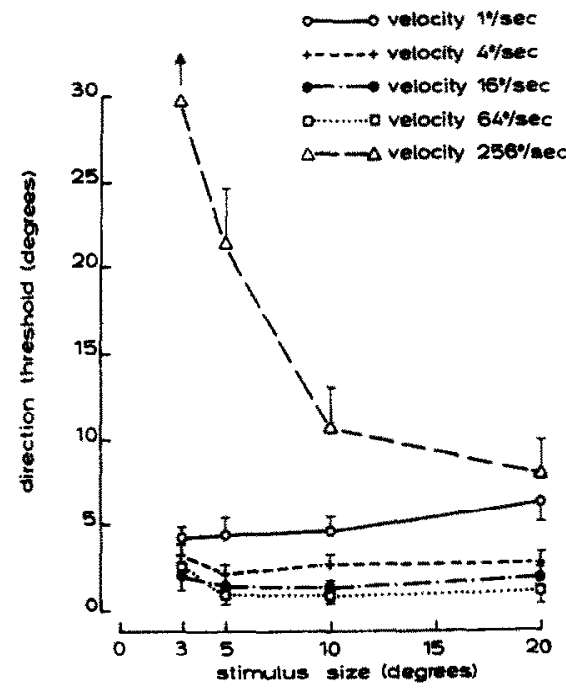

Fig. 7. Direction discrimination thresholds plotted as a function of the size of the random dot pattern. The abscissa refers to the diameter (in degrees) of the circular aperture in which the pattern appeared. Mean data for three subjects.

Vertical bars indicate standard deviations.

velocity for a velocity of $256 \mathrm{deg} \cdot \mathrm{sec}^{-1}$ and a stimulus duration of $50 \mathrm{msec}$. The results are indicated in Fig. 6. This change in stimulus duration simply shifted the JNDs upward for all stimulus sizes.

\section{EXPERIMENT 4: CHANGES IN VELOCITY AND DIRECTION DISCRIMINATION AS A FUNCTION OF STIMULUS DURATION}

The effect of stimulus duration on velocity and direction discrimination was measured for five velocities: $1,4,16,64$ and $256 \mathrm{deg} \cdot \mathrm{sec}^{-1}$. For each velocity and direction discrimination thresholds were determined for stimulus durations ranging from 10 to $200 \mathrm{msec}$. The size of the circular aperture in which the stimulus appeared was $10 \mathrm{deg}$ diameter. Changes in velocity discrimination as a function of stimulus duration are shown in Fig. 8. These results show that at all velocities tested differential velocity sensitivity improves with increasing stimulus duration. The duration at which optimal performance is reached depends however on the reference velocity. For a velocity of $1 \mathrm{deg} \cdot \mathrm{sec}^{-1}$ optimal performance is reached for a duration of 150-200 msec. For velocities ranging from 4 to $64 \mathrm{deg} \cdot \mathrm{sec}^{-1}$ a stimulus duration of $150 \mathrm{msec}$ is needed in order to achieve optimal performance. For a velocity of $256 \mathrm{deg} \cdot \mathrm{sec}^{-1}$ summation is completed for a stimulus duration of $50 \mathrm{msec}$.

Figure 9 shows the effect of stimulus duration on direction discrimination. At all velocities tested, direction discrimination thresholds improve with increasing duration. As is the case for velocity discrimination, the effect of the duration of the motion on direction discrimination is velocity dependent. Direction discrimination thresholds depend more on the duration of the motion for the lowest $\left(1 \mathrm{deg} \cdot \mathrm{sec}^{-1}\right)$ and for the highest $\left(256 \mathrm{deg} \cdot \mathrm{sec}^{-1}\right)$ velocities than for medium velocities. At a velocity of $256 \mathrm{deg} \cdot \mathrm{sec}^{\prime \prime}$, it is unclear whether summation is complete at the largest duration tested (50 msec).

Comparison between velocity discrimination and direction discrimination shows that direction discrimination reaches its optimal value for shorter stimulus durations than velocity discrimination. Comparison of the temporal summation effect shows that for velocities ranging

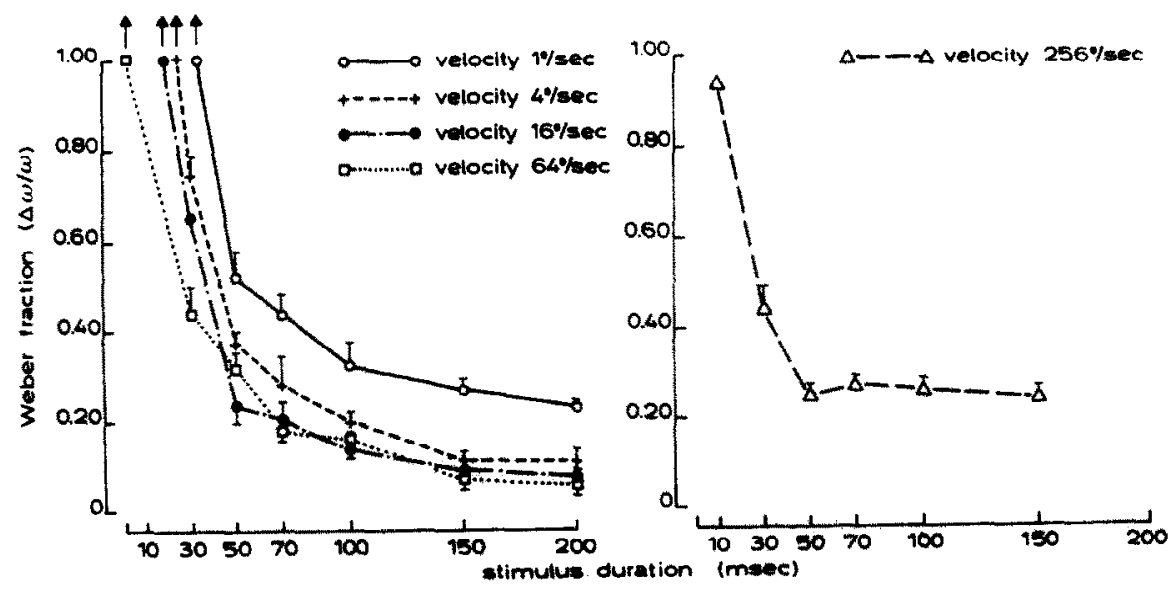

Fig. 8. JNDs in velocity, expressed as Weber-fractions, plotted as a function of stimulus duration for five velocities. The size of the random dot pattern was 10 deg dia. Mean data for three subjects. Vertical bars indicate standard deviations. 


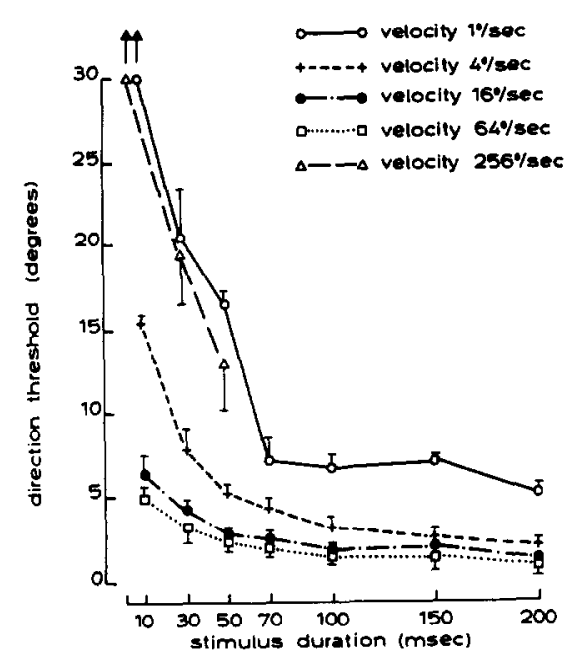

Fig. 9. Direction discrimination thresholds plotted as a function of stimulus duration for five velocities. The size of the random dot pattern was 10 deg dia. Mean data for three subjects. Vertical bars indicate standard deviations.

from 4 to $64 \mathrm{deg} \cdot \mathrm{sec}^{-1} \mathrm{JND}$ in velocity depend more steeply on the stimulus duration than JNDs in direction (Fig. 10).

\section{EXPERIMENT 5: OPPOSED DIRECTION DISCRIMINATION}

In the standard conditions, the threc subjects could perfectly discriminate opposite directions at all velocities. Figure 3 however shows that at low contrast, opposed motion discrimination is only possible at intermediate speeds $\left(2-64 \mathrm{deg} \cdot \mathrm{sec}^{-1}\right)$. Exactly as for the other
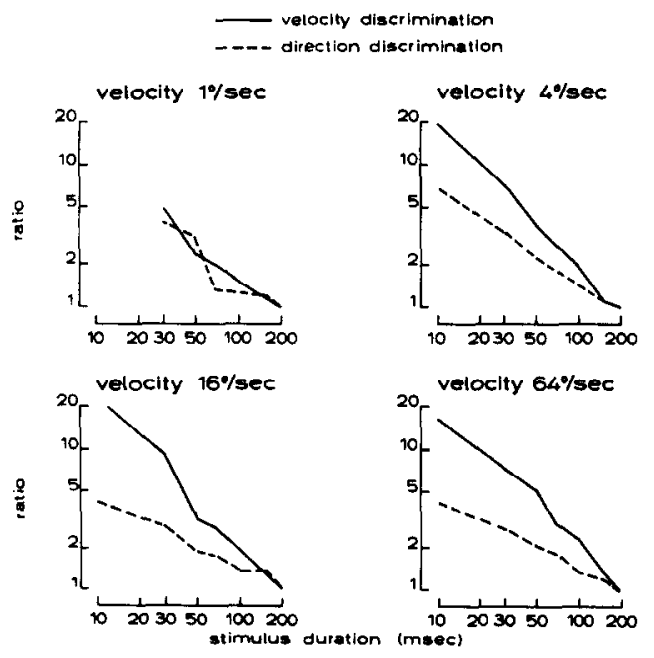

Fig. 10. Comparison between the effect of stimulus duration on velocity discrimination (full line) and direction discrimination (dashed line). In each figure the threshold, normalized according to the lowest threshold, is plotted as a function of stimulus duration, on a double logarithmic scale. two tasks, opposed direction discrimination depended on stimulus size only at $256 \mathrm{deg} \cdot \mathrm{sec}^{-1}$. However only the change in diameter from 3 to $5 \mathrm{deg}$ influenced this performance. Measuring the effect of stimulus duration on opposed direction discrimination showed that almost all conditions yielded a perfect discrimination. Only at I deg $\cdot \mathrm{sec}^{-1}$ the shortest duration tested had a somewhat limiting effect. Hence opposed direction discrimination was far less affected by the stimulus parameters manipulated than the two other tasks.

\section{DISCUSSION}

Our results show that the short range process of human motion perception operates optimally at intermediate speeds. For all the psychophysical tasks studied, JNDs in velocity, JNDs in direction and opposed direction discrimination, optimal performance was obtained for speeds between 4 and $64 \mathrm{deg} \cdot \mathrm{sec}^{-1}$. These findings are in agreement with the hypothesis formulated in our previous studies (Orban et al., 1984, 1986; Orban, 1985), that cortical cells which operate as bandpass filters for velocity (velocity tuned cells) and cortical cells which are selective for the direction of motion, as described in areas 17 and 18 of the cat (Orban et al., 1981a, b) and in area V5 of the monkey (Zeki, 1978; Maunsell and Van Essen, 1983), underly the short range process of human motion perception. Indeed these populations of cells respond maximally at intermediate speeds (Orban et al., 1981a, b; Maunsell and Van Essen, 1983).

Since most cells tuned for velocity are tuned to speeds in the middle range (Orban et al., 1981a; Maunsell and Van Essen, 1983), our linking hypothesis predict a U-shaped relationship between JNDs in velocity, expressed as Weber fractions, and stimulus velocity. This U-shaped relationship has been observed in humans (Orban et al., 1984), cats (Vandenbussche et al., 1986a) and monkeys (Vandenbussche et al., 1986b). In all these experiments a moving light bar was used to study motion perception since this stimulus has also been used to describe the motion characteristics of the cortical cells. Whereas at the single cell level a bar stimulus can be an appropriate stimulus to study motion analysis, at the level of the system as a whole, a random dot pattern is a better way to study the contribution of local motion measurements. While motion of a bar can be detected by the long range process (Braddick, 
1974), the analysis of a random dot pattern motion is based on the short range process. Cortical cells which are analysing visual motion, as revealed in physiological experiments, are supposed to underlie the short range process. The present results show that Weber-fractions in velocity depend in the same way on the stimulus velocity whether a bar or a random dot pattern is used as moving stimulus. These findings therefore support our linking hypothesis. They also show that mechanisms underlying velocity discrimination are not smeared at middle velocities. Finally the present results show that the upper branch of the U-shaped velocity discrimination curve is not due to a decrease in the exposure time nor a limitation in contrast sensitivity at fast velocities. Taken together, the results of our previous studies as well as the present ones show that the U-shaped relation between Weber-fractions in velocity and stimulus velocity is a fundamental relationship, it does not depend on changes in the luminance, the contrast, the length of the barstimulus, it is similar for a bar stimulus and a random dot pattern and it persists for a range of stimulus sizes and stimulus durations.

Our results on direction discrimination extend the observations of Ball and Sekuler (1982, 1987) and of Pasternak and Merigan (1984). Both groups of authors reported an improvement in direction discrimination with increasing velocity up to a critical velocity. Neither of the two studies however investigated direction discrimination at speeds above $20 \mathrm{deg}$. $\mathrm{sec}^{-1}$. Our results are very similar to those of Ball and Sekuler. Converting the $d^{\prime}$ values (Johnson, 1980) of the Ball and Sekuler's (1987) study to $84 \%$ difference thresholds in direction, expressed in degrees, yields a minimal threshold of $1.9 \mathrm{deg}$ which is very similar to the $1.8 \mathrm{deg}$ observed in our study. Also the critical velocity was very similar in both studies: $8-10 \mathrm{deg} \cdot \mathrm{sec}^{\text {i }}$ in Ball and Sekuler's study and $48 \mathrm{deg} \cdot \mathrm{sec}^{-3}$, depending on the subject, in our study. Both the asymptotic threshold $(4.6 \mathrm{deg})$ at intermediate speeds and the critical velocity ( $1-2.2$ $\mathrm{deg} \cdot \mathrm{sec}^{-1}$ ) were different in the Pasternak and Merigan (1984) study. It is not clear how much training the subjects received in the latter study and a restricted training could easily account for the discrepancy.

Considered over a wide range of speeds, JNDs in speed and in direction show a very similar dependence on stimulus velocity. While cortical cells which are bandpass filters for velocity are usually selective for direction, the converse is not true (Orban et al. 1981a, b, 1986). The range of velocities over which cortical cells are direction selective is wider than the range of velocities over which cortical cells are velocity selective. Indeed, as well in the cat as in the monkey, velocity low-pass cells can be direction selective at very low speeds Orban et al, 1981a, b. 1986; Duysens et al. 19871. Therefore. one would expect that the U-shaped function for direction discrimination would be broader than the one for velocity discrimination. This however is not supported by the results. The ratios between the performance at slow velocities and the performance at optimal speed $164 / \mathrm{sec}$ for JNDs in velocity and $32 / \mathrm{sec}$ for JNDs in direction) are larger for direction than for speed discrimination. At $2, \mathrm{sec}$ the ratio is 2 and 3.2 for speed and direction discrimination respectively. while at $1 / \mathrm{sec}$ the atios are 2.5 and 4.4 respectively. It is possible at the extreme speeds a duration cue rather than speed itself is used by the subjects performing speed discrimination just as was the case for a moving light bar (Orban et al. 1984). Such an intrusion is not possible for direction discrimination. Under this assumption, it is possible that in our stimulus conditions the same initial set of detectors is used both for direction and speed discrimination. This is to say that only those cortical cells which are direction as well as velocity selective are involved in the local analysis (the assignment of local motion vectors) of a moving random dot pattern. Cells in area V5 of the monkey are excellent candidates for such a role.

The observation that velocity discrimination is equally good when tested with a light bar than when tested with a random dot pattern indicates that the spatial extent of the pattern itself has little effect. In our previous study (Orban $e t$ al. 1984) we also noted that decreasing the length of the bar stimulus from $7 \mathrm{deg}$ io 1 deg hardly changed velocity discrimination. A spatially extended pattern (in the sense that there are a large number of on off transients or dges) will stimulate more motion detectors than a localized stimulus. The observation that in our experiments the spatial extent of the pattern itself (the comparison bar-random dot pattern) did not influence the JNDs in velocity indicates that the optimal number of motion detectors pooled in order to make fine velocity discriminations is reached for both the bar and the random dot pattern and that the motion signal will not improve as detectors from several local pools 
are recruited. Furthermore a spatially extended pattern which remain visible during the whole stimulus duration will repeatedly stimulate the same motion detectors. Since there were no differences in performance observed between a bar and a random dot pattern, it seems that in velocity and direction discrimination the contribution of integration of signals arising from the same detectors to temporal summation is rather limited.

The results of the temporal and spatial summation experiments confirm that most of these summations reflect simply the recruitment of the detectors from a pool centered on a single retinal locus. The hypothesis that motion detectors can be recruited from a pool as a function of stimulus area and presentation time, was put forward by van Doorn and Koenderink (1982a, b). According to the results of van der Grind et al. (1986), the characteristics of the elementary motion detectors modelled as correlation devices, their delays and spans, are scattered around a mean value. The pooling of the output of elementary motion detectors centered a single retinal locus and tuned to a single velocity (i.e. a single speed and a single direction) but having different characteristics (spans and hence delays) is very attractive from a computational point of view. While the output of an elementary motion detector depends both on stimulus pattern and stimulus velocity, integrating over elements of the same pool reduces dependence on spatial pallern. Furthermore integration over elements of the pool, decreases the probability of false matches to which the detectors with large spans are especially vulnerable. The crucial factor in the recruitment of elements from the pool is the movement traverse which can be limited either by the size of the aperture in which the movement appears or by the stimulus duration. If temporal and spatial summation both reflect recruitment from the local pools of detectors, the ratio between the critical spatial values and the critical temporal value should equal the stimulus velocity. Unfortunately this match can only be checked for the fast velocities used in this study since at $16 \mathrm{deg} \cdot \mathrm{sec}^{-1}$ the smallest aperture tested $(3 \mathrm{deg})$ corresponds to $187 \mathrm{msec}$ which is hardly different from the largest duration tested $(200 \mathrm{msec})$. For direction discrimination the match can only be checked at $64 / \mathrm{sec}$ given the limitations in duration at $256 / \mathrm{sec}$. There is a small improvement in direction discrimination as the stimulus diameter increases from 3 to $5 \mathrm{deg}$. The latter size corrcsponds to a critical duration of $78 \mathrm{msec}$ and indeed direction discrimination levels of between 70 and $100 \mathrm{msec}$. For speed discrimination the match is not as close. For a speed of $256^{\circ} / \mathrm{sec}$ temporal summation is complete at $50 \mathrm{msec}$ which corresponds to $12.8^{\circ}$. Although speed discrimination improves steeply with increasing stimulus diameter up to $10^{\circ}$, it continues to improve somewhat between 10 and $20^{\circ}$. At $64 \% \mathrm{sec}$ speed discrimination does not improve much for diameters over 5 , while it continues to improve for durations beyond $70 \mathrm{msec}$. This latter discrepancy is in keeping with the observation that temporal summation, but not spatial summation, is larger for speed discrimination than for direction discrimination. Thesc results then suggest that while dircetion discrimination only relies on recruitment from the local pool of detectors, an additional factor intervenes in speed discrimination. This could be a purely temporal integration either in the detectors or beyond, or a spatiotemporal factor due to stimulation of longer sequences of neighbouring detectors. In the latter case spatial and temporal summation would not act independently, a prediction which can be tested experimentally.

While JNDs in direction depend strongly on stimulus duration, opposed direction discrimination hardly does. Hence there seems to be very little recruitment of detectors from local pools required for this task. On the other hand at low contrast opposed motion discrimination is only possible at intermediate speeds. Several lines of evidence (Pasternak and Leinen, 1986; Orban et al., 1988) suggest that optimal psychophysical performance at low contrast requires the cooperation of many visual cortical cells. Hence opposed direction discrimination at low contrast requires many elementary detectors. In order to reconcile both conclusions, one has to assume that the detectors used in opposed motion discrimination all have the same span, probably the average span of local pool. That opposed direction discrimination at low contrast is better for intermediate speed than more extreme ones, suggests that the number of elementary detectors with a fixed span (say the average) is larger for intermediate than for extreme velocities. Hence the ensemble of our results can be explained by the following model. (1) For each retinal locus there is a pool of elementary motion detectors tuned to a single velocity (i.e. a speed and a direction). Within a local pool the characteristics of the detectors 
(spans) are scattered around on average and the number of detectors of a given span is larger for pools tuned to intermediate speeds than to extreme speeds. (2) JNDs require the input from many more elementary detectors than opposed motion discrimination and for both types of processing the number of elements required increases with decreasing contrast. (3) In order to compute JNDs elementary motion detectors are recruited from the pool across spans, while for opposed motion discrimination only pooling from detectors with a single span (likely the average of the pool) is used. (4) Spatial summation in speed and direction discrimination relies entirely on recruitment from local pools, while temporal summation relies on recruitment from local pools for direction discrimination but on recruitment and on temporal integration of signals from single detectors in speed discrimination.

In our experiments we measured velocity and direction resolution for stimulation of a single stimulus location. The thresholds we observed are in agreement with those reported in other studies (McKee, 1981; McKee and Welch, 1985; Ball and Sekuler, 1987) and correspond to the results obtained in our previous studies (Orban et al., 1984, 1986). It is interesting to note that the velocity and direction differences needed for a clear segregation by motion (van Doorn and Koenderink, 1983) are extremely large compared with the local velocity and direction resolution levels. The difference in speed required is a Weber fraction of about $100 \%$ and the difference in direction about $30 \mathrm{deg}$. These differences are even larger than the discrimination thresholds for a naive subject first participating in our discrimination experiments. A possible explanation for this difference could be that the segregation studies (van Doorn and Koenderink, 1983) address a different motion analyzing "mechanism". In this respect it is worth noting that from their physiological results, Orban and Gulyas (1988) have postulated the existence of two pathways for the processing of output of cortical cells tuned to a given stimulus parameter such as orientation, speed or direction. In one pathway the local-to-global differences are computed and these signals are used for segregation. While this pathway processes discontinuities in a parameter domain, the second pathway processes the continuous values of the same domain. This latter processing improves the local signals and interpolates between these local values within the limits indicated by the discontinuity pathway. The JNDs in direction and velocity would in this scheme rely on processing in the "continuous" pathway while segregation by speed or direction would rely on the "discontinuity" pathway. The present results and their interpretation given here suggest that the study of JNDs can provide insight in the further processing in the "continuous" pathway in which pooling across elements from local pools is used to give a more reliable and more invariant estimate of the local velocity vector.

\section{REFERENCES}

Allman J, Miczin F. and McGuinness E. (1985) Directionand velocity-specific responses from beyond the classical receptive field in the middle temporal area (MT). Percep. tion 14, 105 126.

Baker C. L. and Cynader M. S. (1986) Spatal receptive-field properties of direction-selective neurons in cat striate cortex. J. Neurophysiol. 55, 11361152

Ball K. and Sekuler R. (1982) A spectic and enduring improvement in visual motion discrimination. Science. N.Y. 218, 697698

Ball K. and Sekuler R. (1987) Direction-specific improvement in motion discrimination. Vision Res: 27, 953-966:

Braddick $O$. (1974) A short range process in apparent motion. Vision Res. 14, 519 527.

De Bruyn B. and Orban G. A. (1986) Human velocity discrimination measured with 2D noise patterns. Perception 15, A8.

van Doorn A. J. and Koenderink J. J. (1982a) Temporal properties of the visual detectability of moving spatial white noise. Expl Brain Res. 45, 179188.

van Doorn A. J. and Koenderink J. (1982b) Spatial properties of the visual detectability of moving spatial white noise. Expl Brain Res. 45, $189-195$.

van Doorn A. J. and Koenderink J. J. (1983) Detectability of velocity gradients in moving random dot patterns. Vision Res. 23, 799804.

Duysens J., Maes H. and Orban G. A. (1987) The velocity dependence of direction selectivity of visual cortical neurons in the cat. J. Physiol., Land, 387, 95-113.

Duysens J., Orban G. A. and Cremieux J. (1985) Velocity selectivity in the cat visual system. II. Independence from interactions between different loci. I. Neurophysiol. 54, 10501067.

van der Grind W.. Koenderink 3, J. and van Doorn A. J. (1986) The distribution of human motion detector properties in the monocular visual field Vision Res. 26, $797-810$

Gulyas B., Orban G. A., Duysens J. and Maes H. (1987) The suppressive influence of moving textured backgrounds on responses of cat striate neurons to moving bars. J. Neurophysiol. 57, 1767-1791

Haddad G. M. and Steinman R. M. (1973) The smallest voluntary saccade: implications for fixation. Vision Res. 13, $1075-1086$.

Hammond P. and Mackay D. M. (1975) Differential responses of cat visual cortical cells to textured stimuli Expl Brain Res. 22, $427-430$. 
Hammond P. and MacKay D. M. (1977) Differential responsiveness of simple and complex cells in cat striate cortex to visual texture. Expl Brain Res. 30, 275-296.

Hassenstein B. and Reichardt W. (1956) Functional structure of a mechanism of percpetion of optical movement. Proc. First Int. Congr. on Cybernetics, Namar, 797-801.

Johnson K. O. (1980) Sensory discrimination: decision process. J. Neurophysiol. 43, 1771-1792.

Julesz B. (1971) Foundations of Cyclopean Perception. University of Chicago Press, Chicago, IL.

Kosnik W.. Fikre J. and Sekuler R. (1985) Improvement in direction discrimination: no role for eye movements. Percept. Psychophys. 38, 554558.

Kowler E. and Steinman R. M. (1979) The effect of expectations on slow oculomotor control. II. Single target displacements. Vision Res. 19, 633-646.

Levitt H. (1971) Transformed up-down methods in psychoacoustics. J. acoust. Soc. Am. 49, 467-477.

Marr D. and Ullman S. (1981) Directional selectivity and its use in early visual processing. Proc. $R$. Soc. Lond. Ser. B. 211, 151-180.

Maunsell J. H. R. and Van Essen C. (1983) Functional properties of neurons in middle temporal visual area of the Macaque monkey. I. Selectivity for stimulus direction. speed and orientation. $J$. Neurophysiol. 49, 1127-1147.

McKee S. P. (1981) A local mechanism for differential velocity detection. Vision Res. 21, 491-500.

Mckee S. P. and Welch L. (1985) Sequential recruitment in the discrimination of velocity. J. opt. Soc. Am. A. 2, 243-251.

Nakayama K. (1985) Biological image motion. Vision Res. 25, 625-660.

Nakayama K. and Tyler C. W. (1981) Psychophysical isolation of movement sensitivity by removal of familiar position cues. Vision Res. 21, 427-433.

Orban G. A. (1985) Velocity tuned cortical cells and human velocity discrimination. In Brain Mechunisms (Edited by Ingle D. J.. Jeannerod M. and Lee D. N.), pp. 371-388. Martinus Nijhoff, Dordrecht.

Orban G. A. and Gulyas B. (1988) Image segregation by motion: cortical mechanisms and implementation in neural networks. In Neural Computers. Series F: Computer and Systems Sciences (Edited by Eckmiller R. and van der Malsburg, C.), pp. 149-158. Springer, Heidelberg.

Orban G. A., Callens M. and Colle J. M. (1975) Unit responses to moving stimuli in area 18 of the cat. Brain Res, 90, 205-219.

Orban G. A.. Kennedy H. and Maes H. (1981a) Response to movement of neurons in areas 17 and 18 of the cat velocity sensitivity. $J$. Neurophysiol. 45, 1043-1058.
Orban G. A.. Kennedy H. and Maes H. (1981b) Response to movement of neurons in areas 17 and 18 of the cat: direction selectivity. J. Neurophysiol. 45, 1059-1073.

Orban G. A., De Wolf J. and Maes H. (1984) Factors influencing velocity coding in the human visual system. Vision Res. 24, 33-39.

Orban G. A., Kennedy H. and Bullier J. (1986) Velocity sensitivity and direction selectivity of neurons in areas $\mathrm{V} 1$ and V2 of the monkey: influence of eccentricity. J. Neurophysiol. 56, $462-480$.

Orban G. A. Vandenbussche E., Sprague I. M. and De Weerd P. (1988) Stimulus contrast and visual cortical lesions. Expl Brain Res. (In press).

Parker A. and Hawken M. (1985) Capabilities of monkey cortical cells in spatial-resolution tasks. J. opt. Soc. Am. A. 2, 1101-1114.

Pasternak T. and Leinen L. (1986) Pattern and motion vision in cats with selective loss of cortical directional selectivity. J. Neurosci. 6, 938-945.

Pasternak T. and Merigan W. H. (1984) Effects of speed on direction discrimination. Vision Res. 24, 1349-1356.

Regan $D$. and Reverley K. I. (1985) Postadaptation orientation discrimination. J. opt. Soc. Am. A. 2, 147-155.

Reichardt W. (1961) Autocorrelation, a principle for the evaluation of sensory information by the central nervous system. In Sensory Communication (Edited by Rosenblith W. A.), pp. 303-317, MIT Press, Cambridge, Mass.

Santen van J. P. H. and Sperling G. (1985) Elaborated Reichardt detectors, J. opt. Soc. Am. A. 2, 300-321.

Siegel S. (1956) Nonparametric statistics for the behavioural Sciences. McGraw-Hill, New York.

Skottun B. C., Bradley A., Sclar G., Ohzawa I. and Freeman R. D. (1987) The effects of contrast on visual orientation and spatial frequency discrimination: a comparison of single cells and behaviour. J. Neurophysiol. 57, 773-786.

Vandenbussche E., Orban G. A. and Maes H. (1986a) Velocity discrimination in the cat. Vision Res. 26, 1835-1849.

Vandenbussche E., Orban G. A. and Maes H. (1986b) Velocity discrimination in the monkey. Soc. Neurosci. Abstr. 12, 1183.

Westheimer G. (1954) Eye movement responses to a horizontally moving visual stimulus. AMA Arch. Ophthal. 52, 932-941.

Wetherill G. B. and Levitt H. (1965) Sequential estimation of points on a psychometric function. Br. J. Math. Statist. Psychol. 18, 1-10.

Zeki S. M. (1978) Functional specialisation in the visual cortex of the rhesus monkey. Nature, Lond. 274, 423-428. 